\title{
Transcutaneous electrical nerve stimulation in the treatment of patients with poststroke urinary incontinence
}

This article was published in the following Dove Press journal:

Clinical Interventions in Aging

23 May 2014

Number of times this article has been viewed

\author{
Zhui-feng Guo',* \\ Yi Liu ${ }^{2, *}$ \\ Guang-hui Hu' \\ Huan Liu' \\ Yun-fei $X u^{\prime}$ \\ 'Department of Urology, ${ }^{2}$ Department \\ of Neurology, Shanghai Tenth People's \\ Hospital, Tongji University, Shanghai, \\ People's Republic of China \\ *These authors contributed equally \\ to this work
}

Correspondence:Yun-fei Xu

30I Middle Yanchang Road Shanghai,

Shanghai Tenth People's Hospital, Shanghai,

200072, People's Republic of China

$\mathrm{Tel}+8602166300588$

Fax +86 02I 6630664 I

Emailyunfeixu_phd@I63.com
Purpose: To investigate the therapeutic effect of transcutaneous electrical nerve stimulation (TENS) on poststroke urinary incontinence (UI).

Patients and methods: Sixty-one patients with poststroke UI were enrolled at the Neurology Department in the Shanghai Tenth People's Hospital of Tongji University between January 2010-January 2011 and were divided into treatment and control groups ( $n=32$ and $n=29$, respectively). TENS was applied to the treatment group, while the control group received basic therapy. The therapeutic group completed the whole set of TENS therapy with a treatment frequency of 30 minutes once a day for 60 days. The positive electrode was placed on the second lumbar spinous process, and the negative electrodes were inside the middle and lower third of the junction between the posterior superior iliac spine and ischia node. The overactive bladder symptom score, Barthel Index, and urodynamics examination were estimated before and after therapy in both groups.

Results: The daily micturition, nocturia, urgent urination, and urge UI in the treatment group significantly improved compared to the control group $(P<0.05)$. The patients in the treatment group were superior in the self-care ability of daily living and also had an advantage over the indexes on maximum cystometry volume, flow rate, and the pressure of detrusor in the end of the filling phase.

Conclusion: TENS improved incontinence symptoms, enhanced the quality of life, and decreased adverse effects; hence, it is recommended in treating poststroke UI.

Keywords: stroke, urinary incontinence, OABSS, Barthel Index, urodynamics, transcutaneous electrical nerve stimulation

\section{Introduction}

A survey carried out by the World Health Organization showed that the People's Republic of China ranked first in the poststroke morbidity rate, which was nearly twice as high as that in the US. Urinary incontinence (UI) is defined as an involuntary leakage of urine, which is an independent risk factor of poststroke prognosis. Overall, the prevalence of UI in hospitalized patients from different countries suggested that between $32 \%-79 \%$ of patients with stroke at admission experience incontinence. ${ }^{1}$ UI seriously affects the life qualities of patients, ${ }^{2}$ and UI costs around $\$ 11$ billion US per year in home nursing in the US. ${ }^{3}$ Nearly 10,000 patients per year needed some form of therapy in Denmark. ${ }^{4}$ Even still, this situation has not received enough consideration in many countries. A survey in Canada showed that only $39 \%$ of occupational therapists and $41 \%$ of physical therapists identified UI after stroke as a problem; and even fewer of them used best-practice assessments and interventions. ${ }^{5}$ 
Transcutaneous electrical nerve stimulation (TENS) treatment is classified as a neuromodulation therapy. Classically, TENS has been used in the management of pain control ${ }^{6}$ and in the treatment of diabetic neuropathy and Raynaud's disease. ${ }^{7}$ So far, several studies have shown positive results about this therapy in the treatment of urinary symptoms. ${ }^{8-12}$ A new study showed that transcutaneous posterior tibial nerve stimulation was safe and acceptable with evidence of potential benefit for bladder dysfunction in elders. ${ }^{13}$ Some studies reported that TENS could be used as a worthwhile treatment for neurological patients with urinary symptoms. ${ }^{8}$ TENS is a relatively convenient, cheap, and noninvasive therapy; it is also free from pharmaceutical side effects. This study prospectively investigated the effect of TENS applied to the special group of patients with poststroke urinary symptoms. It assessed the changes in symptom scores, quality of life, and urodynamic variables.

\section{Patients and methods}

The present study enrolled 61 patients, who were hospitalized from January 2010-January 2011 at the Neurology Department in the Tenth People's Hospital of Tongji University in Shanghai in the People's Republic of China. The inclusion criteria included: patients with poststroke UI; patients who were stable, cooperative, and could effectively communicate (to accomplish therapy); and patients who could finish the survey on urination and pre- and posttreatment. The exclusion criteria included: patients with urinary retention; patients having various reasons for UI in the past (Parkinson's disease, spinal cord disease, dementia, urinary tract tumors, urolithiasis, unhealed urinary tract infections, or history of urinary tract surgery); patients with severe cognitive function disorders (Mini-Mental State Examination ${ }^{14}<22$ points); patients who were unable to communicate effectively; patients with concurrent serious organic diseases; patients who needed massive fluid infusion or usage of drugs that affected urination; and patients who were unable to provide accurate survey information or to complete the treatment period.

The hospital's ethical committee approved this study, and all the patients signed the informed consent form.

Enrolled patients were asked to stop any anticholinergic therapy at least 2 weeks before the first visit. The demographic characteristics and medical history were collected, and a detailed neurological investigation was conducted in the treatment department. The duration of problems, medication history, details of associated impairments with a measure of independence, and details of bowel and bladder management were recorded. Patients then underwent urodynamic analysis (filling rate $20 \mathrm{~mL} /$ minute). After the course of treatment, posttreatment clinical data of patients were collected.

The patients were randomly divided into two groups treatment and control. The treatment group received therapy with TENS (model TENS-21; Homer Ion Laboratory Company Ltd, Tokyo, Japan) for 30 minutes once a day for 60 days, with a pulse duration of $70 \mu \mathrm{S}$ and frequency of $75 \mathrm{~Hz}$ in the form of the unidirectional square wave. The maximum therapeutic current was $16 \mathrm{~mA}(1 \mathrm{k} \Omega)$. According to the nerve innervation, the electrodes' pads were placed as follows: the positive electrode $\left(39 \mathrm{~cm}^{2}\right)$ was on the second lumbar spinous process; and two negative electrodes $\left(30 \mathrm{~cm}^{2}\right)$ were on the inside of the middle and lower third of the junction between the posterior superior iliac spine and the ischia node. Reusable silicone gel electrodes were used in the study, and a small amount of water was placed in the electrodes to make good electrical contact.

The overactive bladder symptom score (OABSS) was obtained using the self-report questionnaire prepared by Homma et $\mathrm{a}^{15}$ to quantify overactive bladder (OAB) symptoms using a 1-week recall period. OABSS measures four broad domains (daily micturition, nocturia, urinary urgency, and urge UI [UUI]). The OABSS score was considered as the sum of the scores from these four domains, which were graded with patients' urination in the latest week. The original OABSS was validated with high reproducibility in a Japanese group of patients with OAB (weighted $\kappa$ coefficients $0.804-1.000$ for each item and 0.861 for OABSS).$^{15}$ The therapeutic efficacy was evaluated in accordance with the OABSS scores obtained before and after treatment. The diagnostic criteria for $\mathrm{OAB}$ were more than 2 points for the urinary urgency and more than 3 points in the total OABSS score; a score of $\leq 5$ points was graded as mild $\mathrm{OAB}, 6-10$ as moderate $\mathrm{OAB}$, and $\geq 12$ as severe $\mathrm{OAB}$ (UI).

Over the past two decades, the Barthel Index (BI) has become well-established, and it acts as the reference standard to validate other scales. ${ }^{16}$ It has concurrent and predictive values, which can be compared with poststroke prognosis. ${ }^{17}$ The content of the BI questionnaire includes ten activities: feeding; bathing; grooming; dressing; bowels; bladder; toilet use; transfers (bed to chair and back); mobility (on level surfaces); and use of stairs. Each item was graded into four classes using 0-20 points, according to whether the patients needed help and which degree of help. The degree of dependency was ranked as: independent (100 points); lightly dependent (75-95); moderately dependent (50-70); severely dependent (25-40); and totally dependent (0-20). 
Table I Demographic and clinical characteristics

\begin{tabular}{|c|c|c|}
\hline Variable & Group I (with TENS) & Group 2 (without TENS) \\
\hline & $(n=32)$ & $(n=29)$ \\
\hline & $M \pm S D$ & $M \pm S D$ \\
\hline \multicolumn{3}{|l|}{ Disease type } \\
\hline Cerebral hemorrhage & 8 & 6 \\
\hline Cerebral infarction & 24 & 23 \\
\hline \multicolumn{3}{|l|}{ Diseased region } \\
\hline Frontal and parietal lobes & 5 & 3 \\
\hline Occipital lobe & 2 & 1 \\
\hline Basal ganglia region & 18 & 19 \\
\hline Cortex multifocal damage & 7 & 6 \\
\hline \multicolumn{3}{|l|}{ Sex } \\
\hline Male & 21 & 21 \\
\hline Female & II & 8 \\
\hline Average age & $(68.1 \pm 7.1) y^{\Delta}$ & $(65.1 \pm 9.8)$ y \\
\hline Average course & $(48.7 \pm 10.5) \mathrm{d}^{\Delta}$ & $(53.1 \pm 18.2) \mathrm{d}$ \\
\hline
\end{tabular}

Note: ${ }^{\triangle} P$-value compared to control group $P>0.05$.

Abbreviations: TENS, transcutaneous electrical nerve stimulation; $M \pm S D$, mean \pm standard deviation; $y, y e a r ; d$, day.

Urodynamic examination was a good way to evaluate the urination for patients with poststroke, and it mainly showed detrusor hyperreflexia ${ }^{18}$ in patients with poststroke. Therefore, detrusor hyperreflexia was decreased when there was an increase in the maximum cystometry capacity or a decrease in the end filling detrusor pressure. The coordination between the function of detrusor and urethral sphincter improved when the flow rate reached the maximum.

Statistical analysis was performed with Statistical Package for Social Sciences, version 13 (SPSS Inc., Chicago, IL, USA). Descriptive analysis was carried out using frequencies, mean, and standard deviations. Enumeration and measurement data were compared between groups using chi-square test and Student's $t$-test, respectively. Associations were considered statistically significant if the $P$ value was $\leq 0.05$.

\section{Results}

The baseline characteristics were similar in both the groups, which included disease types, diseased regions, number, sex, average age, and course (Table 1).
None of the patients had significant adverse effects. The daily micturition, nocturia, urinary urgency, and UUI in the treatment group improved more obviously than in the control group $(P<0.05)$ after 60 days of therapy. The OABSSs in the treatment group were significantly lower than in the control group. Reported in the treatment group were: $17(53.13 \%)$ mild OABs; 12 (37.5\%) moderate OABs; and three $(9.38 \%)$ severe OABs. The control group reported $26(89.66 \%)$ severe OABs and three $(10.34 \%)$ moderate OABs (Table 2).

After 60 days of treatment with TENS, the self-care ability of daily living in the treatment group patients was found to be apparently superior to that in the control group (Table 3). The seven activities - including bowels, bladder, toilet use, transfers (bed to chair and back), bathing, mobility (on level surfaces), and use of stairs - were significantly superior to those in the control group. The BI score was above 50 points for $21(65.63 \%)$ patients in the treatment group, but for only ten (34.48\%) patients in the control group. This clearly showed that TENS improved poststroke incontinence symptoms and activities of daily life.

Table 2 OABSSs of the patients before and after TENS

\begin{tabular}{llllll}
\hline Variable & Group I (with TENS) & & & \multicolumn{2}{c}{ Group 2 (without TENS) } \\
\cline { 2 - 3 } & Before (M \pm SD) & After (M \pm SD) & & Before (M \pm SD) & After (M \pm SD) \\
\hline Daily micturitions & $2 \pm 0.37^{\Delta}$ & $0.67 \pm 0.59^{*}$ & $2 \pm 0.61$ & $1.57 \pm 0.31$ \\
Nocturia & $3 \pm 0.56^{\Delta}$ & $1.13 \pm 0.41^{*}$ & $3 \pm 0.83$ & $2.65 \pm 0.43$ \\
Urgent urination & $5 \pm 0.94^{\Delta}$ & $2.64 \pm 0.98^{*}$ & $5 \pm 0.69$ & $4.09 \pm 0.71$ \\
UUl & $4.02 \pm 0.76^{\Delta}$ & $1.61 \pm 0.32^{*}$ & $4.18 \pm 0.65$ & $3.86 \pm 0.74$
\end{tabular}

Notes: $\triangle P$-value compared to control group $P>0.05$; $* P$-value compared to control group $P<0.05$.

Abbreviations: OABBS, overactive bladder symptom score; TENS, transcutaneous electrical nerve stimulation; $M \pm S D$, mean \pm standard deviation; UUI, urge urinary incontinence. 
Table $3 \mathrm{BI}$ scores of patients before and after TENS

\begin{tabular}{|c|c|c|c|c|}
\hline \multirow[t]{2}{*}{ Variable } & \multicolumn{2}{|c|}{ Group I (with TENS) } & \multicolumn{2}{|c|}{ Group 2 (without TENS) } \\
\hline & Before $(\mathbf{M} \pm \mathbf{S D})$ & After $(\mathbf{M} \pm \mathbf{S D})$ & Before $(\mathbf{M} \pm \mathbf{S D})$ & After $(\mathbf{M} \pm \mathbf{S D})$ \\
\hline Bowels & $0^{\Delta}$ & $6.72 \pm 3.01 *$ & 0 & $2.93 \pm 3.41$ \\
\hline Bladder & $0^{\Delta}$ & $7.97 \pm 2.20 *$ & 0 & $2.24 \pm 3.16$ \\
\hline Grooming & $1.72 \pm 2.43^{\Delta}$ & $6.72 \pm 3.73$ & $1.739 \pm 2.42$ & $3.79 \pm 2.18$ \\
\hline Toilet use & $0^{\Delta}$ & $5.31 \pm 3.80^{*}$ & 0 & $2.24 \pm 3.16$ \\
\hline Feeding & $2.97 \pm 3.56^{\Delta}$ & $8.75 \pm 4.40$ & $2.57 \pm 3.69$ & $5.70 \pm 3.95$ \\
\hline Transfers & $5.47 \pm 4.46^{\Delta}$ & $8.28 \pm 4.14 *$ & $5.5 I \pm 5.23$ & $5.17 \pm 4.53$ \\
\hline Mobility & $3.91 \pm 4.16^{4}$ & $3.91 \pm 2.10^{*}$ & $3.62 \pm 3.76$ & $2.76 \pm 3.43$ \\
\hline Dressing & $2.50 \pm 3.1 I^{\Delta}$ & $5.63 \pm 1.31$ & $2.07 \pm 3.14$ & $3.97 \pm 2.06$ \\
\hline Stairs & $0^{\Delta}$ & $6.36 \pm 3.06 *$ & 0 & $2.24 \pm 3.43$ \\
\hline Bathing & $0^{\Delta}$ & I.72 $\pm 2.4 I^{*}$ & 0 & $0.52 \pm 1.55$ \\
\hline
\end{tabular}

Notes: ${ }^{\triangle} P$-value compared to control group $P>0.05$; * $P$-value compared to control group $P<0.05$.

Abbreviations: $\mathrm{BI}$, Barthel Index; TENS, transcutaneous electrical nerve stimulation; $M \pm S D$, mean \pm standard deviation.

The patients in the treatment group had superior indexes on maximum cystometry volume, maximum flow rate, and the pressure of detrusor at the end of the filling phase than the control group after the 60 days of treatment with TENS (Table 4).

The therapeutic effect on the patients in the treatment group showed that the maximum cystometric capacity and flow rate were evidently greater than at the pretreatment stage. In particular, the decrease in the end filling detrusor pressure showed a greater improvement in the treatment group than in the control group, in which the end filling detrusor pressure only rose slightly.

\section{Discussion}

The rehabilitation of patients with poststroke all around the world is a challenging task. Traditionally, most of the medical institutions in the People's Republic of China mainly pay attention to the rehabilitation of limb function. In recent years, some hospitals have successively started the therapy for activities of daily living and language. The rehabilitation of patients with poststroke UI is still a relatively new topic, and its intervention has not been identified as a problem by therapists even in developed countries. Increasing studies on this topic mainly focus on urodynamic examination and electrical stimulation therapy.
The neuroregulation of urination consists of two nerve centers (supraspinal reflex and spinal reflex centers) and three sets of nerves (sympathetic, parasympathetic, and somatic nerves). These nerves arise from the lateral horn of L1-L4 and S1-S4, and they are distributed in the trigone of the urinary bladder, external urethral sphincter of the bladder neck, and transverse perineus. The conus medullaris (S2-S4), the spinal reflex center of parasympathetic and somatic nerves, plays a leading role in promoting urination among these nerves. This theoretical basis is extremely important in the location of TENS, which has a direct influence on the outcomes. As one of the low frequency electric stimulation therapies, TENS is used extensively in neurological rehabilitation. TENS has been a widely used therapeutic approach in acute and chronic pain syndromes. ${ }^{19,20}$ Wong et al ${ }^{21}$ found that TENS was a convenient and effective therapy for patients with stroke in the rehabilitation of limb function. Hagstroem et $\mathrm{a}^{22}$ suggested TENS could improve refractory daytime incontinence in children with overactive bladder.

In this study of 61 patients with poststroke UI, the basic treatment was effective in improving UI for both groups in different degrees. However, the combination of TENS with the basic therapy achieved significantly superior results than in those treated with the basic therapy alone. The inclusion of TENS brought a significant improvement in UI, which was

Table 4 Urodynamic data of the patients before and after TENS

\begin{tabular}{llllll}
\hline Variable & Group I (with TENS) & & & Group 2 (without TENS) \\
\cline { 2 - 3 } & Before (M \pm SD) & After (M \pm SD) & & Before (M \pm SD) & After (M \pm SD) \\
\hline MCC $(\mathrm{mL})$ & $178 \pm 22^{\Delta}$ & $218 \pm 28^{*}$ & $185 \pm 21$ & $195 \pm 23$ \\
End filling Pdet $\left(\mathrm{cm} \mathrm{H}_{2} \mathrm{O}\right)$ & $57 \pm 11^{\Delta}$ & $49 \pm 16^{*}$ & $59 \pm 9$ & $62 \pm 12$ \\
MFR $(\mathrm{mL} / \mathrm{s})$ & $7.3 \pm 1.4^{\Delta}$ & $10.8 \pm 3.9^{*}$ & $7.1 \pm 1.7$ & $7.4 \pm 1.9$ \\
\hline
\end{tabular}

Notes: $\triangle P$-value compared to control group $P>0.05$; $* P$-value compared to control group $P<0.05$.

Abbreviations: TENS, transcutaneous electrical nerve stimulation; $M \pm S D$, mean \pm standard deviation; MCC, maximum cystometric capacity; Pdet, detrusor pressure; MFR, maximum flow rate. 
a good predictor of stroke severity, not only because of its relationship with infarct size and intracerebral hemorrhage, but also because it might have a more subtle effect on morale and, therefore, influence response to rehabilitation. ${ }^{1}$

The urodynamic data provided quantitative and relatively objective data on the effect of TENS on detrusor function. The changes in detrusor function as perceived by patients were monitored using the OABSS, and the effect of changes in detrusor function on their daily life was monitored using BI.

Most of the results from this study supported an overall improvement in urinary symptoms from TENS, including the urodynamic variables showing a trend toward improvement (Table 4). All the three urodynamic variables changed significantly, which could obviously compromise the bladder function. A number of studies have shown that UI is not only an active predictor of survival but also of recovery. Skeil et $\mathrm{al}^{9}$ applied TENS to 44 neurological patients with urinary symptoms and also achieved the positive outcome of reducing the frequency of micturition, improving bladder function, and reducing potential renal damage. Urodynamic variables that changed significantly included postvoid residual urine and volume of urine leaked. McGuire et $\mathrm{al}^{23}$ also showed that five of the eight patients with miscellaneous neurological disorders, treated with TENS via the posterior tibial or common peroneal nerves, either became dry or improved (reducing the frequency of micturition and improving bladder function); although no urodynamic or statistical data were provided in that study. Hasan et $\mathrm{al}^{24}$ also provided conservative treatments using TENS and S3 neuromodulation to patients with severe refractory detrusor instability, and the results revealed significant changes in urodynamic parameters and presenting symptoms.

As demonstrated by the OABSS and BI scores, there are significant changes in detrusor function and quality of life from the use of TENS for these patients, which should be a key to expand the indication of this therapy to treat patients with poststroke UI. For poststroke patients, incontinence was a marker for stroke severity because of its association with death and disability and its influence on the place of discharge of poststroke survivors. ${ }^{25,26}$ The decreasing of urinary frequency - especially the episodes of nocturia - could reduce risks of many falls and their consequences. ${ }^{27}$ Strong evidence for an improvement in quality of life after TENS was provided by the BI, where there were significant improvements in bladder and bowel continence, toileting, transfers, mobility, use of stairs, and bathing, which all could obviously improve life for both the patients and their caregivers.
In the treatment of patients with poststroke UI, other therapies, such as acupuncture therapy and medium frequency electrotherapy, are also available. However, these patients are mostly aged, ${ }^{1}$ and a significant number of them have a high prevalence for diabetes or with metallic implants in vivo. Acupuncture therapy would damage skin, and medium frequency electrotherapy would be contraindicated for the patients within metal internal fixation. In accordance with the principle of minimally invasive treatments, TENS is remarkably more noninvasive than acupuncture therapy and medium frequency electrotherapy. The noninvasive aspect of this form of treatment was particularly important in this group of patients.

Even though the results obtained in the current study are encouraging, the outcomes presented are only for a shorter term. It is important to know the necessity of new treatments or even repetition of therapy over time. Hence, trials with longer follow-up periods are recommended to confirm the long-term curative effect.

In conclusion, TENS improved urinary symptoms and quality of life and decreased adverse effects in this group of patients with poststroke UI. TENS can be recommended in this clinical condition; however, more studies with a larger sample size and longer follow-up are mandatory to validate the study results.

\section{Disclosure}

The authors report no conflicts of interest in this work.

\section{References}

1. Brittain KR, Peet SM, Castleden CM. Stroke and incontinence. Stroke. 1998;29(2):524-528.

2. Edwards DF, Hahn M, Dromerick A. Poststroke urinary loss, incontinence and life satisfaction: when does post-stroke urinary loss become incontinence? Neurourol Urodyn. 2006;25(1):39-45.

3. Bean JF, Kiely DK, Cairns KD, Morris JN. Influence of poststroke urinary incontinence on disability: the nursing home setting. Am J Phys Med Rehabil. 2003;82(3):175-181.

4. Tibaek S, Gard G, Jensen R. Is there a long-lasting effect of pelvic floor muscle training in women with urinary incontinence after ischemic stroke? A 6-month follow-up study. Int Urogynecol J Pelvic Floor Dysfunct. 2007;18(3):281-287.

5. Dumoulin C, Korner-Bitensky N, Tannenbaum C. Urinary incontinence after stroke: identification, assessment, and intervention by rehabilitation professionals in Canada. Stroke. 2007;38(10):2745-2751.

6. Long DM. Fifteen years of transcutaneous electrical stimulation for pain control. Stereotact Funct Neurosurg. 1991;56(1):2-19.

7. Kaada B. Vasodilation induced by transcutaneous nerve stimulation in peripheral ischemia (Raynaud's phenomenon and diabetic polyneuropathy). Eur Heart J. 1982;3(4):303-314.

8. Cooperberg MR, Stoller ML. Percutaneous neuromodulation. Urol Clin North Am. 2005;32(1):71-78, vii.

9. Skeil D, Thorpe AC. Transcutaneous electrical nerve stimulation in the treatment of neurological patients with urinary symptoms. BJU Int. 2001;88(9):899-908. 
10. Congregado Ruiz B, Pena Outeiriño XM, Campoy Martínez P, León Dueñas E, Leal López A. Peripheral afferent nerve stimulation for treatment of lower urinary tract irritative symptoms. Eur Urol. 2004;45(1): 65-69.

11. Amarenco G, Ismael SS, Even-Schneider A, et al. Urodynamic effect of acute transcutaneous posterior tibial nerve stimulation in overactive bladder. J Urol. 2003;169(6):2210-2215.

12. Yamanishi T, Kamai T, Yoshida K. Neuromodulation for the treatment of urinary incontinence. Int J Urol. 2008;15(8):665-672.

13. Booth J, Hagen S, McClurg D, et al. A feasibility study of transcutaneous posterior tibial nerve stimulation for bladder and bowel dysfunction in elderly adults in residential care. J Am Med Dir Assoc. 2013;14(4): $270-274$.

14. Grut M, Fratiglioni L, Viitanen M, Winblad B. Accuracy of the MiniMental Status Examination as a screening test for dementia in a Swedish elderly population. Acta Neurol Scand. 1993;87(4):312-317.

15. Homma Y, Yoshida M, Seki N, et al. Symptom assessment tool for overactive bladder syndrome - overactive bladder symptom score. Urology. 2006;68(2):318-323.

16. Collin C, Wade DT, Davies S, Horne V. The Barthel ADL Index: a reliability study. Int Disabil Stud. 1988;10(2):61-63.

17. Kong KH, Chua KS, Tow AP. Clinical characteristics and functional outcome of stroke patients 75 years old and older. Arch Phys Med Rehabil. 1998;79(12):1535-1539.

18. Nitti VW, Adler H, Combs AJ. The role of urodynamics in the evaluation of voiding dysfunction in men after cerebrovascular accident. J Urol. 1996;155(1):263-266.
19. Somers DL, Somers MF. Treatment of neuropathic pain in a patient with diabetic neuropathy using transcutaneous electrical nerve stimulation applied to the skin of the lumbar region. Phys Ther. 1999;79(8): 767-775.

20. Lampl C, Kreczi T, Klingler D. Transcutaneous electrical nerve stimulation in the treatment of chronic pain: predictive factors and evaluation of the method. Clin J Pain. 1998;14(2):134-142.

21. Wong AM, Su TY, Tang FT, Cheng PT, Liaw MY. Clinical trial of electrical acupuncture on hemiplegic stroke patients. Am J Phys Med Rehabil. 1999;78(2):117-122.

22. Hagstroem S, Mahler B, Madsen B, Djurhuus JC, Rittig S. Transcutaneous electrical nerve stimulation for refractory daytime urinary urge incontinence. J Urol. 2009;182(4 Suppl):2072-2078.

23. McGuire EJ, Zhang SC, Horwinski ER, Lytton B. Treatment of motor and sensory detrusor instability by electrical stimulation. J Urol. 1983; 129(1):78-79.

24. Hasan ST, Robson WA, Pridie AK, Neal DE. Transcutaneous electrical nerve stimulation and temporary $\mathrm{S} 3$ neuromodulation in idiopathic detrusor instability. J Urol. 1996;155(6):2005-2011.

25. Nakayama H, Jorgensen HS, Pedersen PM, Raaschou HO, Olsen TS. Prevalence and risk factors of incontinence after stroke. The Copenhagen Stroke Study. Stroke. 1997;28(1):58-62.

26. Greveson GC. Long-term outcome following stroke. Age Ageing. 1993; 22(6):477.

27. Dubeau CE. The aging lower urinary tract. J Urol. 2006;175(3 Pt 2): S11-S15.
Clinical Interventions in Aging

\section{Publish your work in this journal}

Clinical Interventions in Aging is an international, peer-reviewed journal focusing on evidence-based reports on the value or lack thereof of treatments intended to prevent or delay the onset of maladaptive correlates of aging in human beings. This journal is indexed on PubMed Central, MedLine,

\section{Dovepress}

CAS, Scopus and the Elsevier Bibliographic databases. The manuscript management system is completely online and includes a very quick and fair peer-review system, which is all easy to use. Visit http://www.dovepress. com/testimonials.php to read real quotes from published authors. 